\title{
An Image-based Approach for 3D Left Atrium Functional Measurements
}

\author{
Alan Morris ${ }^{1}$, Eugene Kholmovski ${ }^{3}$, Nassir Marrouche ${ }^{4}$, Joshua Cates ${ }^{5}$, Shireen Elhabian ${ }^{1,2}$ \\ ${ }^{1}$ Scientific Computing and Imaging Institute, University of Utah, Salt Lake City, UT, USA \\ ${ }^{2}$ School of Computing, University of Utah, Salt Lake City, UT, USA \\ ${ }^{3}$ Department of Radiology and Imaging Sciences, School of Medicine, \\ University of Utah, Salt Lake City, UT, USA \\ ${ }^{4}$ Tulane University School of Medicine, New Orleans, LA, USA \\ ${ }^{5}$ Division of Cardiovascular Medicine, School of Medicine, \\ University of Utah, Salt Lake City, UT, USA
}

\begin{abstract}
There is growing interest in the assessment of function of the left atrium (LA) in patients with atrial fibrillation $(A F)$. Existing methods of LA functional measurement only quantify a limited subset of the functional parameters from a single or biplane CINE-MRI scan through the LA. Here, we propose an image-based method for comprehensive evaluation of the function of the entire LA in $3 D .4 D$ LA images were reconstructed from a series of CINE image stack covering the whole LA with small or no gap between thin slices. A segmentation from a high-resolution Magnetic Resonance Angiography (MRA) was registered and propagated through pairwise deformable registrations covering the cardiac cycle. Volume, LA ejection fraction and surface strain were computed for each timepoint and registered to Late Gadolinium Enhancement (LGE) scans for each of 52 patient scans. A correlation coefficient of -0.11 was calculated between LGE and strain, indicating that fibrotic tissue correlates with reduced elasticity.
\end{abstract}

\section{Introduction}

Understanding both structural and functional changes in the LA has become fundamental to understanding AF disease burden and progression. CMR imaging has proven to be a useful tool in visualizing and quantifying both structural and functional remodeling as part of AF disease progression. Different CMR sequences are employed to address both of these types of measurements. LGE imaging is used to non-invasively quantify disease progression in the form of left atrial fibrosis [1]. CINE sequences demonstrate cardiac motion, but are typically limited to one or two planes going through the heart.

LA fibrosis detected from an LGE sequence has been found to be associated with history of stroke, hypertension and heart failure. It has also been shown to predict recurrence following AF ablation as well as major adverse cardiovascular and cerebrovascular events [2,3].

Functional measurements of the LA have been shown to predict AF in the general population and additionally provide predictive capability for cardiovascular events, cardiomyopathy, ischemic heart disease, and valvular heart disease [4-6]. Left atrial function can be quantified by speck-tracking echocardiography, but this method is limited by the thin walls of many of the areas of the LA. Newer methods of evaluating LA function using CMR have recently been developed [7]. However, these existing CMR methods are limited in that they are only able to quantify functional parameters from a single or biplane CINE MRI scan through the LA.

In this study, we propose a novel image-based method for a more comprehensive evaluation of the function of the entire LA using fully 3D methods. We combine image processing techniques such as deformable image registration as well as surface mesh-based techniques to evaluate both localized surface motion as well as overall global functional measurements.

\section{Methods}

Cohort: 52 cardiac MRI scans of 43 patients were acquired using the following protocol. 32 were pre-ablation, 20 were post ablation. 1,351 CINE time points were captured with an average of $26 \mathrm{CINE}$ time points per patient.

CMR Acquisition: Cardiac MR (CMR) studies were performed on either a 1.5 or 3 Tesla MR scanner (Siemens Healthcare, Erlangen, Germany). This study included CINE CMR, contrast enhanced (CE) MRA, and 3D LGE. CINE CMR was acquired in either axial or standard 4chamber orientation using an ECG-gated, balanced steadystate free precision (bSSFP) sequence. Typical scan pa- 
rameters for 1.5T CINE CMR were: echo time $(\mathrm{TE})=1.2$ $\mathrm{ms}$, repetition time $(\mathrm{TR})=2.7 \mathrm{~ms}$, flip angle $(\mathrm{FA})=70^{\circ}$, contiguous slices with 6-8 $\mathrm{mm}$ thickness, and typical spatial and temporal resolution of $1.6 \times 1.6 \mathrm{~mm}$ and $45 \mathrm{~ms}$, respectively.

The corresponding scan parameters for 3T CINE CMR were $\mathrm{TE} / \mathrm{TR}=1.5 / 3.5 \mathrm{~ms}, \mathrm{FA}=45^{\circ}$, contiguous slices with $6 \mathrm{~mm}$ thickness, and typical spatial and temporal resolution of $1.3 \times 1.3 \mathrm{~mm}$ and $35 \mathrm{~ms}$, respectively. CE-MRA was performed during slow contrast infusion using a 3D saturation recovery-prepared, respiration-navigated, ECGgated, gradient echo pulse sequence. The scan parameters were: axial imaging volume with field of view $(\mathrm{FOV})=$ $400 \times 400 \times 110 \mathrm{~mm}$ and voxel size of $1.25 \times 1.25 \times 2.5$ $\mathrm{mm}$. The other scan parameters for 1.5T CE-MRA were: saturation time $(\mathrm{TI})=150 \mathrm{~ms}, \mathrm{TE} / \mathrm{TR}=1.6 / 4.4 \mathrm{~ms}, \mathrm{FA}=$ $20^{\circ}$. The corresponding scan parameters for 3T CE-MRA were $\mathrm{TI}=120 \mathrm{~ms}, \mathrm{TE} / \mathrm{TR}=1.3 / 2.8 \mathrm{~ms}, \mathrm{FA}=17^{\circ} .3 \mathrm{D}$ LGE scan was initiated 15-20 minutes after contrast injection using a $3 \mathrm{D}$ inversion recovery-prepared, respirationnavigated, ECG-gated, gradient echo pulse sequence. The scan parameters were: axial imaging volume with FOV $=$ $400 \times 400 \times 110 \mathrm{~mm}$ and voxel size of $1.25 \times 1.25 \times 2.5$ $\mathrm{mm}$. The other scan parameters for 1.5T LGE-MRI were: $\mathrm{TE} / \mathrm{TR}=2.7 / 5.4 \mathrm{~ms}, \mathrm{FA}=20^{\circ}$. The corresponding scan parameters for 3T LGE-MRI were: TE/TR $=1.4 / 3.1 \mathrm{~ms}$, $\mathrm{FA}=14^{\circ}$. A small subset of data were acquired during the diastolic phase of the LA cardiac cycle to minimize LA wall blurring in CE-MRA and 3D LGE scans.

Computational Pipeline: For each patient, we combined the three types of image sequences. A cardiac imaging expert performed a manual segmentation of the LA anatomy from a high-resolution MRA as well as the LGE image. LA fibrosis was quantified using established methods [1]. 4D LA CMR images were reconstructed from a series of CINE image stacks covering the entire LA with small or no gap between slices. Individual 3D volumes were extracted and reconstructed for each time point of the cardiac cycle from the 4D image.

To aid in image processing and registration steps, a region of interest was defined on each sequence around the left atrium. For the LGE and MRA, the LA segmentation plus some padding was used to automatically crop the images to the region of interest. For the CINE time points, a $\mathrm{K}$-means clustering approach was used to identify the areas of motion within the image and crop to these areas. This makes the registration processes focus on the deformations of the myocardium rather that outside structures.

The anatomical segmentation was registered to the closest time point from the extracted 3D time points. Registration was performed using the ANTS toolkit [8]. To verify registration accuracy, a manual segmentation was performed on the individual time point volumes of seven patients, with 185 3D segmentations. An average DICE over- lap of $85.6 \%$ was calculated between the manual time point segmentations and the MRA segmentations registered to the time points. Pairwise 3D deformable registrations were performed between each consecutive time point to create a continuous set of deformable transforms mapping through the entire cardiac cycle.

A 3D polygonal surface mesh was constructed from the MRA segmentation in the original MRA coordinate space using the marching cubes algorithm. The surface mesh was then transformed through the transformation defined by the MRA and closest CINE time point. Each of the paired time point deformations was then applied, one at a time to transform the mesh through each time point. This process results in a high density point-to-point correspondence between every time point.

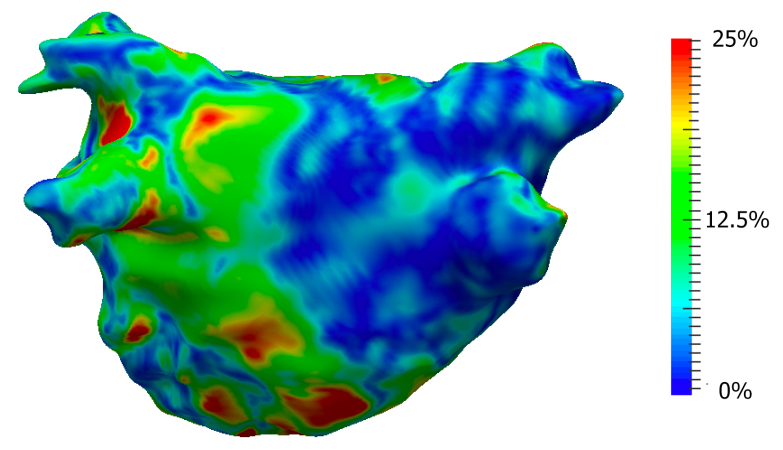

Figure 1. 3D Surface Strain Map. The color map shows the maximum lagrangian strain at each point on the surface over the cardiac cycle.

Using this set of matched surface meshes, we are able to calculate a variety of functional measurements. The volume of the LA is easily calculated at each time point in the cardiac cycle using existing methods [9]. With the volume calculated at each time point, we then calculate the Left Atrial Ejection Fraction (LAEF) using equation 1.

$$
L A E F=\frac{\left(L A V_{\max }-L A V_{\min }\right)}{L A V_{\max }}
$$

Since the surface meshes computed at each time point have full correspondence across time points, we can track a given vertex and its neighbors through the cardiac cycle. We can compute the Euclidean displacement, which is the absolute distance (millimeters) that a point moves from its starting position.

Lagrangian strain is defined by the deformation of an object relative to its original length (e.g. [10]. Traditional CMR techniques on a single plane CINE rely on feature tracking of pixels within the single slice [11]. Such methods are not able to account for cardiac motion that is not 


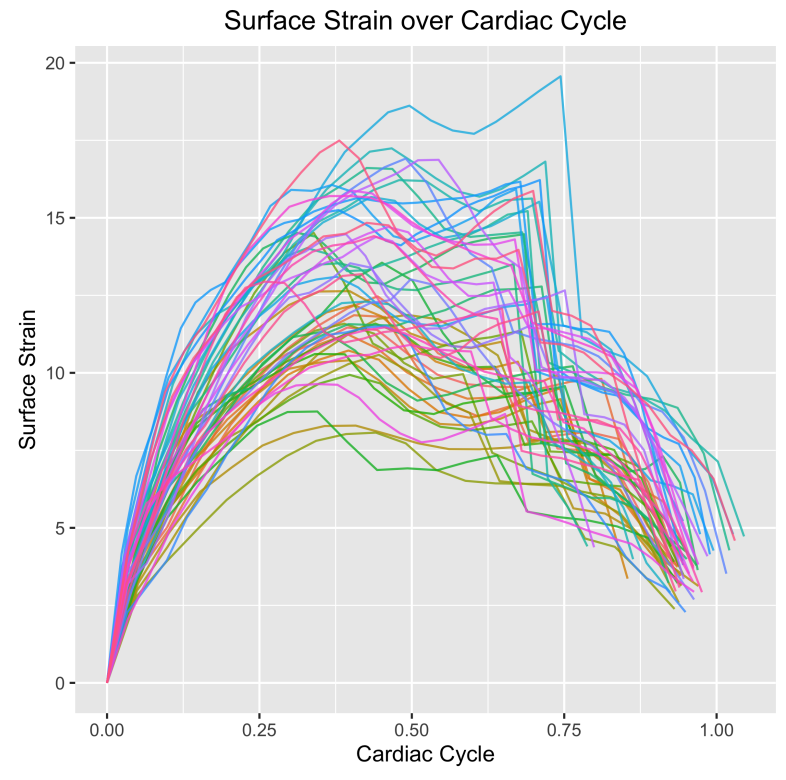

Figure 2. Average Surface Strain for each patient over the parameterized (0-1) cardiac cycle

in-plane with the orientation of the CINE image. One particular advantage of our approach is that our deformation calculations are performed on full 3D volumes from one time point to the next and are able to account for motion in any direction.

We define the term surface strain as a Lagrangian measure of deformation along the surface. With a regular triangular surface mesh of near uniform spacing, vertex neighbors provide a 3D measure of original edge length between neighbors. Areas that expand during the cardiac cycle will see their mesh edge lengths increase relative to the initial length and this is calculated as the surface strain for a given vertex (average of neighboring edge length deformations). This is in contrast to displacement because a given vertex could be displaced by a significant amount without actually deforming significantly from its neighbors. For example, this could happen where a section of dense scar moves significantly in Euclidian space due to stretching in other parts of the LA wall. Our computation of surface strain accounts for this, and little to no strain will be present at the site of the dense scar. See Figure 1 for an example 3D surface strain map. These calculated 3D maps were then registered to LGE maps to correlate per-point functional measurements with fibrotic tissue characterization. LGE values were quantified and given as a normalized value above the quantified threshold.

\section{Results}

3D strain maps were generated for each time point for each of 52 patient scans. Volume and strain curves were

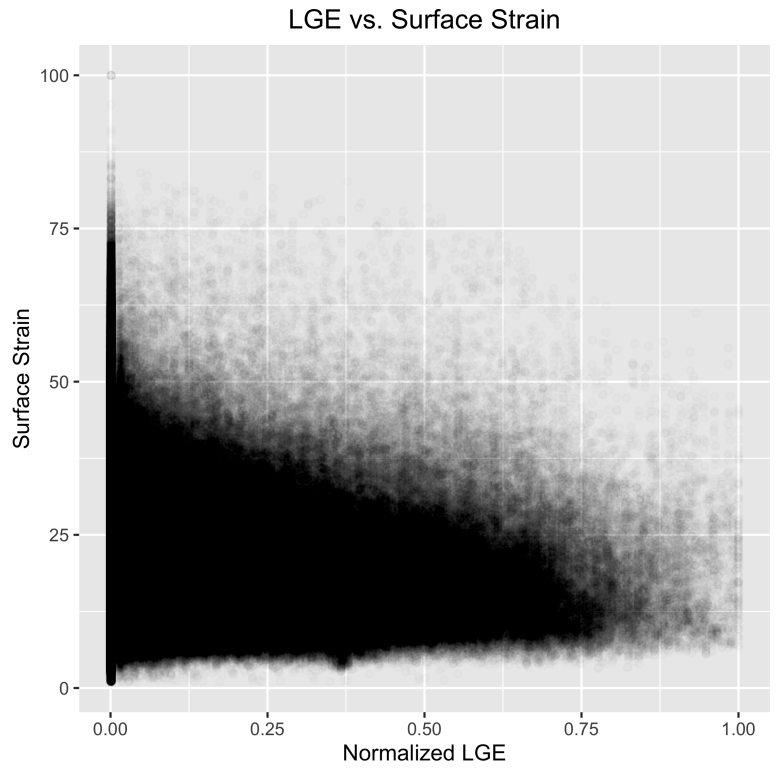

Figure 3. Maximum Surface Strain for each mesh vertex vs normalized LGE intensity

aggregated for each scan. See Figures 2 and 4. LAEF was calculated to be $27.5 \% \pm 10.2 \%$. Peak global surface strain, the 3D analog of longitudinal strain, was calculated to be $12.3 \% \pm 2.6 \%$ over all of the patients.

A point-by-point analysis of $1,748,352$ points across each surface mesh for each patient allows us to investigate localized relationships between structure and function. At each point, values for LGE, strain, and displacement are calculated. A correlation coefficient of -0.11 was computed between LGE and strain ( $\mathrm{p} ; 0.001)$, indicating that fibrotic tissue correlates with reduced elasticity in the LA wall. See Figure 3.

Peak surface strain was compared between pre-ablation and post-ablation scans, and the difference was found to be statistically significant. The mean for pre-ablation was 13.97 and the mean for post ablation was 12.18 ( $\mathrm{p}=$ $0.01208)$.

\section{Limitations and Future Work}

This method of analysis requires a full stack of CINE images through the LA. This is not part of a normal cardiac imaging study, so further adoption of this approach is unfortunately not as easy as applying it to typically-acquired sequences. Also, for this reason, the available cohort of patients was limited to those prospectively acquired. Due to the recent nature of the scans, we do not yet have outcome data for these patients to test the predictive capability of these analyses. As soon as these data become available, we plan to perform such studies. 


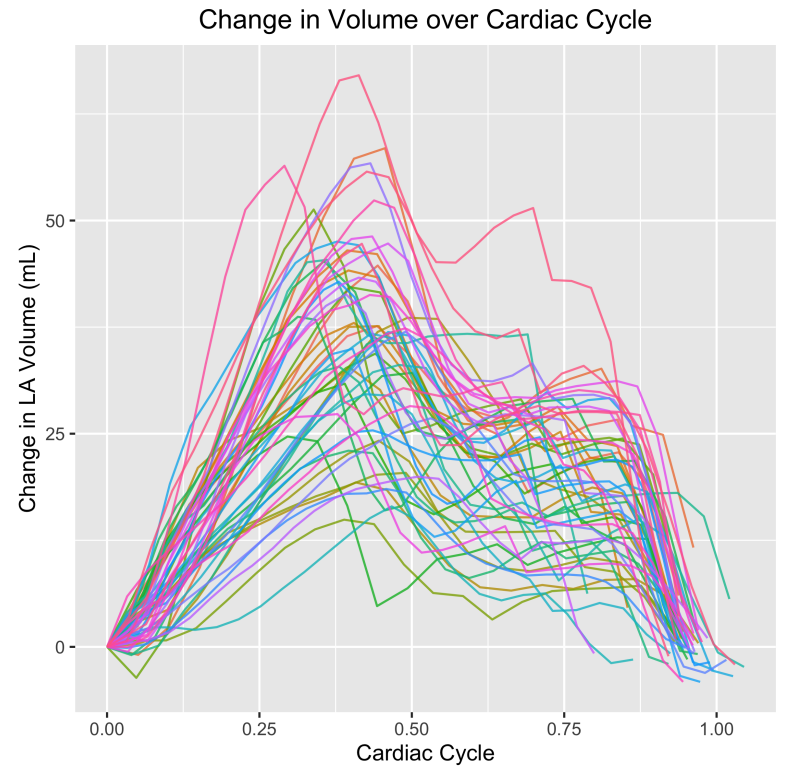

Figure 4. Volume over parameterized cardiac cycle

\section{Conclusion}

A novel method for fusing and evaluating structural and functional 3D MRI through time using only a single segmentation has been described. This fully 3D assessment of function may offer advantages over traditional 2D approaches that are limited to evaluation in only 1-2 slices. Additionally, the available 3D fibrosis maps can be more easily correlated against a full 3D strain map to investigate and show the relationship between structure and function. Further studies will determine if there is increased prognostic value in $3 \mathrm{D}$ functional assessment with regard to outcomes such as AF recurrence and stroke.

\section{Acknowledgments}

This work was supported by the National Institutes of Health under grant numbers NIBIB-U24EB029011, NIAMS-R01AR076120, and NHLBI-R01HL135568. The content is solely the responsibility of the authors and does not necessarily represent the official views of the National Institutes of Health.

\section{References}

[1] Oakes RS, et al. Detection and quantification of left atrial structural remodeling with delayed-enhancement magnetic resonance imaging in patients with atrial fibrillation. Circulation Apr 2009;119(13):1758-1767.

[2] King JB, Azadani PN, Suksaranjit P, Bress AP, Witt DM, Han FT, Chelu MG, Silver MA, Biskupiak J, Wilson BD, Morris AK, Kholmovski EG, Marrouche N. Left Atrial
Fibrosis and Risk of Cerebrovascular and Cardiovascular Events in Patients With Atrial Fibrillation. J Am Coll Cardiol Sep 2017;70(11):1311-1321.

[3] Marrouche NF, et al. Association of atrial tissue fibrosis identified by delayed enhancement MRI and atrial fibrillation catheter ablation: the DECAAF study. JAMA Feb 2014;311(5):498-506.

[4] Badano LP, et al. Standardization of left atrial, right ventricular, and right atrial deformation imaging using twodimensional speckle tracking echocardiography: a consensus document of the EACVI/ASE/Industry Task Force to standardize deformation imaging. Eur Heart J Cardiovasc Imaging 06 2018;19(6):591-600.

[5] Olsen FJ, et al. Relationship between left atrial functional measures and incident atrial fibrillation in the general population. JACC Cardiovascular Imaging 2019;12(6):981-989. ISSN 1936-878X.

[6] Hoit BD. Left atrial size and function: role in prognosis. J Am Coll Cardiol Feb 2014;63(6):493-505.

[7] Inoue YY, et al. Quantitative tissue-tracking cardiac magnetic resonance (CMR) of left atrial deformation and the risk of stroke in patients with atrial fibrillation. J Am Heart Assoc Apr 2015;4(4).

[8] Avants BB, Tustison NJ, Song G, Cook PA, Klein A, Gee JC. A reproducible evaluation of ANTs similarity metric performance in brain image registration. Neuroimage Feb 2011;54(3):2033-2044.

[9] Alyassin AM, Lancaster JL, Downs JH, Fox PT. Evaluation of new algorithms for the interactive measurement of surface area and volume. Med Phys Jun 1994;21(6):741752.

[10] Hoit BD. Strain and strain rate echocardiography and coronary artery disease. Circ Cardiovasc Imaging Mar 2011; 4(2):179-190.

[11] Hor KN, Baumann R, Pedrizzetti G, Tonti G, Gottliebson WM, Taylor M, Benson DW, Mazur W. Magnetic resonance derived myocardial strain assessment using feature tracking. J Vis Exp Feb 2011;(48).

Address for correspondence:

Alan Morris, 72 S Central Campus Drive Room 3750, Salt Lake City, Utah 84112, amorris@ sci.utah.edu

Disclosure: E. Kholmovski has equity interest in Marrek, Inc. and has received consultant's fees from Marrek, Inc. Morris, J. Cates report equity interest in Marrek Inc. N. Marrouche received consulting fees from Abbott, Biotronik, Wavelet Health, Cardiac Design, Medtronic, Preventice, Vytronus, Biosense Webster, Marrek, Inc, and Boston Scientific; research funding from Abbott, Boston Scientific, GE Healthcare, Siemens, Biotronik, Vytronus, and Biosense Webster; ownership interest in Marrek, Inc, and Cardiac Designs; and has conducted contracted research with Biosense Webster, Medtronic, St. Jude Medical/Abbott, and Boston Scientific. 